\title{
CREDIBILITY AND PERSISTENCY
}

\author{
BY VIRGINIA R. YOUNG \\ University of Wisconsin-Madison
}

\begin{abstract}
Policyholders often decide to buy, renew, or cancel insurance based on the premium charged by the insurer compared with what they expect their claims will be. It is important for actuaries to consider the persistency of policyholders because the financial well-being of the insurer depends on spreading its risk over a large book of business. We use statistical decision theory to develop premium formulas that account for the past experience of a given policyholder, the experience of the entire collection of policyholders, and the likelihood of the policyholder renewing with or buying from a given insurer, that is, persistency.

We assume that the persistency of policyholders depends on the arithmetic difference between the premium charged and their anticipated claims. We extend the work of TAYLOR (1975) in which he obtains linear credibility formulas by minimizing loss functions that incorporate the persistency of policyholders. We consider Taylor's loss functions and other objective functions, including those that account for the amount of business the insurer writes or renews.
\end{abstract}

\section{KEYWORDS}

Credibility; persistency; statistical decision theory.

\section{INTRODUCTION}

It is important for actuaries to consider the persistency of policyholders because the financial well-being of the insurer depends upon long-term profitability and upon spreading its risk over a large book of business. An insurer also wishes to retain business because writing initial business is more expensive than renewing existing business. We develop premium formulas that account for the past experience of a given policyholder, the experience of the entire collection of policyholders, and the likelihood of the policyholder renewing with or buying from a given insurer, that is, persistency.

The framework under which we determine the effect of the persistency of policyholders on premiums is statistical decision theory and its application to credibility theory. Credibility theory seeks to find systematic methods for calculating a policyholder's insurance premium based on that policyholder's past experience and the experience of the entire group of policyholders. Current formulas ASTIN BULLETIN, Vol. 26, No. 1, 1996, pp. 53-69 
in credibility theory often calculate premium as a weighted sum of the average experience of the policyholder and the average experience of the entire collection of policyholders. In order to avoid an off-balance from the application of credibility, these formulas usually require that a policyholder will renew no matter what premium is charged.

We assume that the persistency of policyholders depends on the arithmetic difference between the premium charged and their anticipated claims. Considering the arithmetic difference makes sense because we assume that the policyholders are the same size (see Section 2.1). If one wishes to model the persistency of policyholders of different sizes, one might assume that the persistency of policyholders depends on the relative difference, instead of the arithmetic difference. We develop credibility formulas that optimize functions that consider the amount of business that an insurer writes, as well as the monetary gain of the insurer. We, thus, extend the work of TAYLOR (1975) in which the obtains linear credibility formulas by minimizing loss functions that incorporate the persistency of policyholders.

SUNDT (1983) also considers the effect of persistency in credibility rating. His approach differs from ours in that he assumes that the likelihood of renewing is not affected by the premium charged by the insurer. Instead, he assumes that the persistency of the policyholder gives the insurer information about the claim distribution of the policyholder.

We review the work of BüHLMANN (1967, 1970) and TAYlor (1975) in Section 2. Bühlmann derives a credibility formula by minimizing the expected value of a squared-error loss function. Similarly, Taylor minimizes the expected value of the monetary loss to the insurer while discounting the loss by the persistency of policyholders. In Section 3, we introduce objectives that an insurer might consider optimizing.

We propose an exponential persistency function in Section 4 and develop a general credibility formula in Section 5. In Sections 6 and 7, we calculate credibility formulas in two parametric cases--normal-normal and Poisson-gamma. Finally, we suggest future research in Section 8.

\section{WORK OF BÜHLMANN AND TAYLOR}

\subsection{Notation and Assumptions}

Assume that the total claims of a given policyholder, or risk, in the $i^{\text {th }}$ policy period (usually one year), is a random variable $X_{i} \mid(\Theta=\theta)$, or more simply, $X_{i} \mid \theta, i=$ $1, \ldots, n$. For a given value $\Theta=\theta$, assume that the random variables $X_{i} \mid \theta, i=$ $1, \ldots, n$, are independently and identically distributed according to a conditional probability (density) function $f(x \mid \theta)$. Assume that the value $\theta$ is fixed for a given risk, although it is generally unknown. For existing policyholders, denote the probability (density) function of $\Theta$ by $\pi(\theta)$, also called the structure function (BühlmanN, 1970). Note that we tacitly assume that the policyholders are the same size because the distribution of the total claims of a policyholder selected at random is given by the marginal distribution of $X$. 
Suppose the insurer has $n$ years of claim experience for a policyholder: $\boldsymbol{x}=$ $\left\langle x_{1}, \ldots, x_{n}\right\rangle \in X^{n}$. In this work, we consider credibility formulas that are not necessarily linear, denoted by $d(\boldsymbol{x})$, in which $d$ is a real-valued function on $X^{n}$. We also consider linear credibility formulas of the form $a+b \bar{x}$, in which $a$ and $b$ are constants to be determined and $\bar{x}$ is the arithmetic means of the claims $x_{1}, \ldots, x_{n}$. We refer to $d(\boldsymbol{x})$ as the renewal premium, or simply premium, for year $n+1$, and we are not loading for administrative expenses.

For a prospective policyholder, $d(\boldsymbol{x})$ is more accurately termed the initial premium based on past experience of the risk, but we blur this distinction because the insurance products that typically use credibility premiums are annually renewable ones. For this reason, sales personnel must often "sell" the policy annually even if the policyholder is renewing and not initially buying.

\subsection{Work of Bühlmann}

To estimate the future claims of a risk, $X_{n+1} \mid \theta$, with unknown $\theta$, BüHLMANN (1967, 1970) minimizes the expected value of the squared-error loss function

$$
L\left(E\left[X_{n+1} \mid \theta\right], d(\boldsymbol{x})\right)=\left(E\left[X_{n+1} \mid \theta\right]-d(\boldsymbol{x})\right)^{2} .
$$

Under our assumptions, the resulting optimal premium $d^{*}(\boldsymbol{x})$ is

$$
E\left[X_{n+1} \mid x\right]=\int E\left[X_{n+1} \mid \theta\right] \pi(\theta \mid x) d \theta .
$$

By restricting the form of the renewal premium $d(x)$ to be a linear combination of the claim experience, $x_{1}, \ldots, x_{n}$, and by using the same squared-error loss function, BühLMANN $(1967,1970)$ obtains the following credibility formula

$$
d^{*}(\boldsymbol{x})=(1-Z) E[X]+Z \bar{x},
$$

in which $E[X]=E_{\theta} E[X \mid \theta]$ is the overall mean; $Z=n /(n+k)$; and $k=$ $E_{\theta}[\operatorname{Var}[X \mid \theta]] / \operatorname{Var}_{\theta}[E[X \mid \theta]]$. The numerator of $k$ is called the expected process variance; the denominator, the variance of the hypothetical means.

In certain cases, the premium $E\left[X_{n+1} \mid \boldsymbol{x}\right]$ is linear and, thus, equals the linear credibility formula. JeWELL $(1974 a, 1974 b)$ verifies conditions under which this exact credibility occurs. Also, please refer to WILLMOT (1994; Chapter 4) in which he clearly explains the foregoing theory and illustrates it by providing many examples.

\subsection{Work of Taylor}

One of the properties that $d^{*}(\boldsymbol{x})=E\left[X_{n+1} \mid \boldsymbol{x}\right]$ satisfies is that the sum of premiums over the portfolio of risks equals the expected claims from the portfolio (BüHLMANN, 1967). In confirming this property, Bühlmann implicitly assumes that the structure of the portfolio does not change as a result of the rating formula. TAYLOR (1975) challenges this assumption and asserts that if a policyholder tends to cancel when it is renewed with a premium that is higher than its anticipated 
claims, then the premium income will not necessarily equal the expected claims. He proposes a loss function that explicitly accounts for the decision of policyholders to buy, cancel, or renew their insurance. He suggests that the insurer minimize its expected financial loss while discounting for the persistency of policyholders.

Taylor defines a persistency function $p(\theta, x, d(x))$ to be the ratio of the exposure in year $n+1$ to the exposure in year $n$ for the risk class given by $\Theta=\theta$ with $n$-year claim experience $\boldsymbol{x}$ and premium $d(\boldsymbol{x})$ for year $n+1$. The exposure in year $n$ is the number of existing policyholders. To be somewhat rigorous for a continuous structure parameter $\Theta$, think of $p$ as an "instantaneous" ratio or a density function.

His loss function is the financial loss, discounted by persistency,

$$
L\left(\theta, x_{1}, a+b x_{1}\right)=p\left(\theta, x_{1}, a+b x_{1}\right)\left(E\left[X_{2} \mid \theta\right]-\left(a+b x_{1}\right)\right),
$$

and he finds values for $a$ and $b$ that minimize the expected loss. Note that he restricts $n=1$ and $d(\boldsymbol{x})=a+b x_{1}$.

Taylor assumes that $p$ is a linear function of the arithmetic difference between what the insurer charges, $a+b x_{1}$, and the amount of claims the policyholder expects to incur. He then considers two cases: In the first, Taylor calls the policyholder unbiased because the policyholder expects to incur $E\left[X_{2} \mid \theta\right]$, its hypothetical mean; in the second, the policyholder is biased and expects to incur $x_{1}$, its recent claim experience. (Note that Taylor does not use the term biased in a statistical sense because the expected value of $x_{1}$ is $E[X \mid \theta]$.) His two persistency functions are, thus,

Unbiased risk:

$$
\begin{aligned}
& p\left(\theta, x_{1}, a, b\right)=1-e\left(a+b x_{1}-E\left[X_{2} \mid \theta\right]\right) \\
& p\left(\theta, x_{1}, a, b\right)=1-e\left(a+b x_{1}-x_{1}\right)
\end{aligned}
$$

Biased risk:

in which $e$ is a positive constant; Taylor calls the parameter $e$ a price-elasticity of exposure.

The linear credibility premiums that Taylor finds are

$$
\begin{array}{ll}
\text { Unbiased risk: } & (1-Z) E[X]+Z x_{1}+1 /(2 e) ; \\
\text { Biased risk: } & \left(1-Z^{*}\right) E[X]+Z^{*} x_{1}+1 /(2 e) ;
\end{array}
$$

In these formulas, $Z$ is the Bühlmann credibility weight, and $Z^{*}=1 / 2(1+Z)>Z$. Note that in each case, the credibility premium is a weighted average of $x_{1}$ and $E[X]$, plus a flat load, $1 /(2 e)$, independent of the claim distribution or the policyholder's experience. One can consider this load a risk charge for the policyholder selecting against the insurer.

\section{OBJECTIVES OF THE INSURANCE COMPANY}

\subsection{Maximize Underwriting Gain and Amount of Business}

One of the goals of an insurance company, as for any company, is to earn a profit. The profit or underwriting gain is the excess of income over outgo. Insurance 
premiums contain provisions for claims (including risk margins) and for the expenses of administering the insurance policy. Outgo consists of claims, expenses, and experience rating refunds (MOREWOOD, 1992).

In this work, we consider the component of the underwriting gain equal to the excess of the provision for claims and risk in the premium over the claims themselves. In other words, we ignore service fees, investment income, loadings for expenses, as well as the expenses themselves, and experience rating refunds.

Another possible goal of an insurance company is to increase its book of business. MOREWOOD (1992) notes that writing new business depends on competitive premium rates and that renewing existing business depends on how fair the policyholder perceives the price. He points out that underwriting gain and growth are interdependent: Rapid growth and large profit margins in the premiums are usually inversely related. We, therefore, propose finding a function $d^{*}: X^{n} \rightarrow \mathrm{R}$ such that the combination

$$
\begin{aligned}
U G+h B & =E[p(\theta, \boldsymbol{x}, d(\boldsymbol{x}))(d(\boldsymbol{x})-E[X \mid \theta])]+h E[p(\theta, \boldsymbol{x}, d(\boldsymbol{x}))] \\
& =E[p(\theta, \boldsymbol{x}, d(\boldsymbol{x}))(d(\boldsymbol{x})+h-E[X \mid \theta])]
\end{aligned}
$$

is maximum when $d=d^{*}$. The parameter $h$ is a non-negative constant, and we take the expectation with respect to the joint distribution of $X_{1}, \ldots, X_{n}$, and $\Theta$. In Section 4 , we propose a specific formula for the persistency function $p$, but here it is any real-valued function defined on $\Theta \times X^{n} \times R$. Recall that $p$ accounts for initial business as well as renewal.

The first term on the right hand side in equation (3.1) is the expected value of the underwriting gain discounted for the persistency of policyholders. We write $U G$ to denote this first term. The second term is $h$ multiplied by the expected relative amount of business written, or $B$. It is reasonable to constrain $U G \geq 0, B \geq 1$, or both. If we let $h$ approach 0 , then by maximizing $U G+h B$, we maximize the expected underwriting gain $U G$; if we let $h$ approach $\infty$, then we maximize the expected amount of business written $B$.

The parameter $h$ converts the relative amount of business into monetary units (see equation (5.1) below). To choose its values, an actuary may wish to consider the potential loss or gain of revenue to cover fixed administrative expenses. Also, one may choose $h$ according to one of the following criteria:

- $h \geq 0$ is the smallest value such that $B \geq M$, for some $M \geq 1$, in which $B$ is evaluated at the optimal $d^{*}$.

- $h \geq 0$ is the largest value such that $U G \geq M$, for some $M \geq 0$, in which $U G$ is evaluated at the optimal $d^{*}$.

In this context, one can think of $h$ or $1 / h$ as a Lagrange multiplier in a constrained optimization problem. At the end of Section 6.2, we apply these criteria in a hypothetical example. 


\subsection{Optimize Properties of the Book of Business}

An insurance company may set goals concerning the structure of its book of business. If the structure of the company's business is given by $\pi(\theta)$ during year 1 , and the credibility premium for year 2 is $d\left(x_{1}\right)$, then the expected structure of the business in year 2 is described by

$$
\pi_{2}(\theta)=\kappa \int p\left(\theta, x_{1}, d\left(x_{1}\right)\right) f\left(x_{1} \mid \theta\right) \pi(\theta) d x_{1},
$$

in which $k$ is a normalizing constant.

One goal may be to ensure that the grand mean decreases from year to year. This occurrence indicates that the company is writing risks with lower expected claims. The grand mean in year 2 , expected at time 0 , is

$$
\mu_{02}=\kappa \iint x_{2} p\left(\theta, x_{1}, d\left(x_{1}\right)\right) f(\boldsymbol{x} \mid \theta) \pi(\theta) d x d \theta,
$$

in which $\boldsymbol{x}=\left\langle x_{1}, x_{2}\right\rangle$. Note that $\mu_{02}$ is the expected value of $X_{2}$, with respect to the distribution $\int f\left(x_{2} \mid \theta\right) \pi_{2}(\theta) d \theta$.

Another goal may be to ensure that the total variance decreases over time. This occurrence may enable actuaries to price more accurately by making claims more predictable. The variance in year 2 , expected a time 0 , is

$$
\begin{aligned}
E\left[\left(X_{2}-\mu_{02}\right)^{2}\right]= & E_{\theta}\left(E\left[X_{2}^{2} \mid \theta\right]-E\left[X_{2} \mid \theta\right]^{2}\right)+\left(E_{\theta}\left[E\left[X_{2} \mid \theta\right]^{2}\right]-\mu_{02}^{2}\right) \\
= & \text { Expected Process Variance + Variance of Hypothetical } \\
& \text { Means, }
\end{aligned}
$$

in which $\Theta$ is distributed according to $\pi_{2}(\theta)$. We examine these two goals in two parametric cases in Sections 6 and 7.

\section{PERSISTENCY}

We assume, as does TAYLOR (1975), that persistency depends on the arithmetic difference between premium charged and anticipated claims. Such an assumption may be suitable because we assume that risks are the same size. In our work, we explicitly account for Taylor's belief that policyholders most likely expect claims somewhere between $E[X \mid \theta]$ and $\bar{x}$. We do so by expressing the policyholder's anticipated claims as a linear combination of $E[X \mid \theta]$ and $\bar{x}$, namely, $(1-c) E[X \mid \theta]+c \bar{x}, 0 \leq c \leq 1$. The difference between what the insurer charges and what the policyholder expects is, therefore,

$$
\Delta=d(\boldsymbol{x})-[(1-c) E[X \mid \theta]+c \bar{x}] \text {. }
$$

Note that when $n=1$, the amount that the policyholder expects to incur, $(1-c) E\left[X_{2} \mid \theta\right]+c x_{1}$, includes as special cases the two that Taylor examines. For unbiased risks, $c=0$; for biased risks, $c=1$.

Taylor points out one major weakness of the linear persistency functions that he uses: They may take on negative values, implying that the amount of business is negative. We, therefore, propose an exponential persistency function

$$
p(\Delta)=\delta \exp (-\lambda \Delta)
$$


in which $\delta>0$ and $\lambda>0$. Such a function $p$ is always positive and behaves as one expects, namely, it decreases as $\Delta$ increases, and conversely. The parameter $\delta$ is the relative amount of business written if the insurer charges what the policyholder expects its claims will be, and $\lambda$ measures the sensitivity of policyholders to the difference $\Delta$. That is, $\lambda$ is analogous to the parameter $e$ of Taylor, the priceelasticity of exposure.

We assume that $\delta, \lambda$, and $c$ (the relative weight the policyholder gives its claim experience) are fixed for all risks. These assumptions are perhaps unrealistic because one expects that for a given $\Delta$, an existing policyholder is more likely to renew than a prospective one to buy. One also may argue that policyholders buy insurance from a particular company based on that company's premium relative to premiums offered by other insurance companies in the market. To adapt our persistency function to that model, one could replace the difference $\Delta$ with the following

$$
\Delta^{\prime}=d(\boldsymbol{x})-d_{\text {market }}(\boldsymbol{x}),
$$

in which $d_{\text {market }}$ is the (lowest) premium charged by the market. One might also use a dynamic version of this model to explain the underwriting cycle experienced in many lines of insurance.

\section{UNCONSTRAINED MAXIMIZATION OF $U G+h B$}

The combination of underwriting gain and relative amount of business, $U G+h B$, includes $U G$ as a special case by setting $h=0$ and includes $B$ by letting $h$ approach $\infty$. We, therefore, do not work through the details of maximizing $U G$ or $B$ separately. Instead, we maximize $U G+h B$ as given in equation (3.2) and obtain the following theorem.

Theorem 5.1: Let $X_{i} \mid \theta, i=1, \ldots, n$, be independent and identically distributed random variables given $\Theta=\theta$. Let $\mathscr{F}$ be the set of functions $d: X^{n} \rightarrow \mathrm{R}$ for which the gain function

$$
G=\delta \exp \{-\lambda(d(x)-[(1-c) E[X \mid \theta]+c \bar{x}])\}(d(x)+h-E[X \mid \theta])
$$

is integrable with respect to the joint distribution of $X_{1}, X_{2}, \ldots, X_{n}$ and $\Theta$. The expected value of $G$ is maximized when $d=d^{*}$, with $d^{*}$ given by

$$
d^{*}(x)=1 / \lambda-h+\left.D_{t}\left\{\left.\ln E_{\theta}\right|_{x}[\exp \{t E[X \mid \theta]\}]\right\}\right|_{t=\lambda(1-c)},
$$

provided $d^{*}$ is in $\mathscr{F}$. Here $D_{t}$ denotes the operator of differentiation with respect to $t$.

Proof : For $d$ in $\mathscr{F}$, we apply Fubini's theorem to switch the order of integration in the expectation of $G$. The expected value of $G$ is, therefore, maximized when we maximize

$$
\left.E_{\theta}\right|_{x}[\delta \exp \{-\lambda(d(x)-(1-c) E[X \mid \theta]-c \bar{x})\}(d(x)+h-E[X \mid \theta])],
$$


for an arbitrary sample $\boldsymbol{X}=\boldsymbol{x}$, in which we take the expectation with respect to the posterior distribution of $\Theta \mid \boldsymbol{x}$. Treating expression (5.2) as a function of $d(\boldsymbol{x})$, and applying standard techniques from calculus, we find that the maximum occurs when

$$
\begin{aligned}
& \left.\lambda d^{*}(\boldsymbol{x}) E_{\theta}\right|_{x}[\exp \{\lambda(1-c) E[X \mid \theta]\}]= \\
& =\left.\lambda d^{*}(\boldsymbol{x}) E_{\theta}\right|_{x}[E[X \mid \theta] \exp \{\lambda(1-c) E[X \mid \theta]\}] \\
& \quad+\left.(1-\lambda h) E_{\theta}\right|_{x}[\exp \{\lambda(1-c) E[X \mid \theta]\}],
\end{aligned}
$$

or, after solving for $d^{*}(\boldsymbol{x})$,

$$
\begin{aligned}
d^{*}(\boldsymbol{x})= & 1 / \lambda-h+E_{\theta}[E[X \mid \theta] \exp \{\lambda(1-c) E[X|\theta|\}] \\
& \div\left. E_{\theta}\right|_{x}[\exp \{\lambda(1-c) E[X \mid \theta]\}] \\
= & 1 / \lambda-h+\left.D_{t}\left\{\ln E_{\left.\theta\right|_{x}}[\exp \{t E[X \mid \theta]\}]\right\}\right|_{t=\lambda(1-c)}
\end{aligned}
$$

One may interpret the terms in equation (5.1) as follows: The first, $1 / \lambda$, is a flat load similar to the one found by Taylor in equations $(2.3 \mathrm{a}, \mathrm{b})$, namely, $1 /(2 e)$; it partially accounts for the sensitivity of policyholders to the premium charged. The second term, $-h$, offsets for how much the insurer weights the relative amount of business, $B$. Note that $d^{*}(\boldsymbol{x})$ decreases one unit for every unit of increase of $h$; therefore, the more we weight $B$, the more we decrease the optimal premium, as one might expect. The third term is an Esscher premium (GERBER, 1980); it equals $a(\boldsymbol{x})$ that minimizes the expected value of the following loss function:

$$
(a(x)-E[X \mid \theta])^{2} \exp \{\lambda(1-c) E[X \mid \theta]\} .
$$

\section{NORMAL-NORMAL}

\subsection{Unconstrained Maximization of $U G+h B$}

To maximize $U G+h B$ in the normal-normal case, we apply Theorem 5.1 to obtain the following proposition.

Proposition 6.1 : Let $X_{i} \mid \theta \sim N\left(\theta, \sigma^{2}\right), i=1, \ldots, n$, be independent and identically distributed normal random variables, with unknown mean $\theta$ and known variance $\sigma^{2}>0$. Let $\Theta \sim N\left(\mu, \tau^{2}\right)$, with known mean $\mu$ and variance $\tau^{2}>0$. Then $U G+h B$ is maximized when

$$
d^{*}(\boldsymbol{x})=1 / \lambda-h+\{(1-Z) \mu+Z \bar{x}\}+\lambda \tau^{2}(1-Z)(1-c),
$$

in which $Z=n \tau^{2} /\left(n \tau^{2}+\sigma^{2}\right)$, the Bühlmann credibility weight.

Proof: The posterior distribution of $\Theta \mid x$ is normal with mean

$$
\mu^{*}=(1-Z) \mu+Z \bar{x}
$$

and variance 


$$
\left(\tau^{2}\right)^{*}=\sigma^{2} \tau^{2} /\left(n \tau^{2}+\sigma^{2}\right)=\tau^{2}(1-Z)
$$

Because $E[X \mid \theta]=\theta$, substitute the moment generating function of $\Theta \mid \boldsymbol{x}$ into equation (5.1). The moment generating function of a normal random variable $\Theta \sim N\left(\mu, \tau^{2}\right)$ is

$$
M_{\theta}(t)=E_{\theta}[\exp \{\theta t\}]=\exp \left\{\mu t+\tau^{2} t^{2} / 2\right\} .
$$

The derivative, with respect to $t$, of the natural logarithm of this moment generating function is

$$
\mu+\tau^{2} t
$$

To calculate the credibility formula in equation (5.1), substitute $\mu^{*}$ from equation (6.2) for $\mu,\left(\tau^{2}\right)^{*}$ from equation (6.3) for $\tau^{2}$, and $\lambda(1-c)$ for $t$ in equation (6.5). We obtain

$$
\begin{aligned}
d^{*}(\boldsymbol{x}) & =1 / \lambda-h+\left(\mu^{*}+\left(\tau^{2}\right)^{*} \lambda(1-c)\right) \\
& =1 / \lambda-h+\{(1-Z) \mu+Z \bar{x}\}+\lambda \tau^{2}(1-Z)(1-c) .
\end{aligned}
$$

Note that $d^{*}(\boldsymbol{x})$ is a linear function of the average claim experience $\bar{x}$; therefore, we have a type of exact credibility in this case. This credibility premium is the sum of five interesting terms. We discuss the first two at the end of Section 5 ; they occur in $d^{*}(\boldsymbol{x})$ in general. The sum of the third and fourth terms is the standard Bühlmann credibility estimate in the normal-normal case. The fifth expression, depends on how much the policyholders weight their own claim experience relative to their true mean.

To see how the optimal premium $d^{*}(x)$ changes when the parameter $\lambda$ changes, examine

$$
D_{\lambda} d^{*}(\boldsymbol{x})=-1 / \lambda^{2}+\tau^{2}(1-Z)(1-c) .
$$

Observe that if $c$ is sufficiently close to 1 (that is, the policyholders weight their claim experience heavily) or if $n$ is sufficiently large, then $d^{*}(x)$ decreases as $\lambda$ increases. In this case, as the policyholders become more sensitive to the arithmetic difference $\Delta$, the lower the optimal premium.

\subsection{Constrained Maximization of $U G+h B$}

Up to this point, we have not constrained the values of $U G$ and $B$. It is reasonable to require that $U G \geq 0, B \geq 1$, or both; in other words, the insurer does not lose money, business, or both. We examine two sets of constraints and obtain the following propositions.

Proposition 6.2: Given the assumptions in Proposition 6.1 and the restrictions $U G \geq 0$ and $d(x)=a+b \bar{x}$, we maximize $U G+h B$ when $d=d^{*}$, with $d^{*}$ given by

$$
d^{*}(x)=1 / \lambda-h+\{(1-Z) \mu+Z \bar{x}\}+\lambda \tau^{2}(1-Z)(1-c)
$$


for $h \leq 1 / \lambda$, and

$$
d^{*}(\boldsymbol{x})=\{(1-Z) \mu+Z \bar{x}\}+\lambda \tau^{2}(1-Z)(1-c),
$$

for $h>1 / \lambda$.

Proof : $U G+h B=E[\delta \exp \{-\lambda(a+b \bar{x})-(1-c) \theta-c \bar{x}\}(a+h+b \bar{x}-\theta)]$

$$
\begin{aligned}
(6.8)= & \delta \exp \{-\lambda a\}(a+h) E_{\theta}\left[\exp \{\theta \lambda(1-c)\} E_{X \mid \theta}[\exp \{-\lambda(b-c) \bar{x}\}]\right] \\
& +\delta \exp \{-\lambda a\} b E_{\theta}\left[\exp \{\theta \lambda(1-c)\} E_{X} \mid{ }_{\theta}[x \exp \{-\lambda(b-c) \bar{x}\}]\right] \\
& -\delta \exp \{-\lambda a\} E_{\theta}\left[\theta \exp \{\theta \lambda(1-c)\} E_{X} \mid{ }_{\theta}[\exp \{-\lambda(b-c) \bar{x}\}]\right]
\end{aligned}
$$

Use $M_{X} \mid{ }_{\theta}(t)$ and $\left.E_{X}\right|_{\theta}[x \exp \{x t\}]$ to calculate the expected values in $U G+h B$.

More specifically,

$$
\begin{aligned}
E_{\boldsymbol{X} \mid \theta}[\exp \{\bar{x} t\}] & =\left\{M_{X \mid \theta}(t / n)\right\}^{n} \\
& =\exp \left\{\theta t+\sigma^{2} t^{2} /(2 n)\right\}
\end{aligned}
$$

and

$$
\begin{aligned}
E_{X \mid \theta}[\bar{x} \exp \{\bar{x} t\}] & =\left\{M_{X \mid \theta}(t / n)\right\}^{n-1} E_{X \mid \theta}[x \exp \{x t / n\}] \\
& =\exp \left\{\theta t+\sigma^{2} t^{2} /(2 n)\right\}\left[\theta+\sigma^{2} t / n\right]
\end{aligned}
$$

Substitute these expressions into $U G+h B$, equation (6.8), to obtain

$$
\begin{aligned}
& U G+h B= \delta \exp \{-\lambda a\}(a+h) E\left[\exp \left\{\lambda(1-b) \theta+\lambda^{2}(b-c)^{2} \sigma^{2} /(2 n)\right\}\right]+ \\
&+\delta \exp \{-\lambda a\} b E\left[\exp \left\{\lambda(1-b) \theta+\lambda^{2}(b-c)^{2} \sigma^{2} /(2 n)\right\} \times\right. \\
&\left.\times\left[\theta-\lambda(b-c) \sigma^{2} / n\right]\right]-\delta \exp \{-\lambda a\} \times \\
& \times E\left[\theta \exp \left\{\lambda(1-b) \theta+\lambda^{2}(b-c)^{2} \sigma^{2} /(2 n)\right\}\right] \\
&= \delta \exp \left\{-\lambda a+\lambda^{2}(b-c)^{2} \sigma^{2} /(2 n)\right\} \times \\
& \times\left\{\left(a+h-\lambda b(b-c) \sigma^{2} / n\right) E[\exp \{\theta \lambda(1-b)\}]-(1-b) \times\right. \\
&\times E[\theta \exp \{\theta \lambda(1-b)\}]\} \\
&(6.9)=\delta \exp \left\{-\lambda a+\lambda^{2}(b-c)^{2} \sigma^{2} /(2 n)+\lambda(1-b) \mu+\lambda^{2}(1-b)^{2} \tau^{2} / 2\right\} \\
& \times\left\{a+h-\lambda b(b-c) \sigma^{2} / n-(1-b) \mu-\lambda(1-b)^{2} \tau^{2}\right\} .
\end{aligned}
$$

By setting $h=0$ in equation (6.9), we see that

(6.10) $U G \geq 0$ if and only if $a \geq \lambda b(b-c) \sigma^{2} / n+(1-b) \mu+(1-b)^{2} \tau^{2}$.

The values of the parameters $a$ and $b$ that appear in $d^{*}(x)$ in equation (6.1) satisfy the inequality (6.10) if and only if $h \leq 1 / \lambda$. Therefore, if $h \leq 1 / \lambda$, then the insurance company does not expect to lose money by using the credibility formula $d^{*}(x)$ in equation (6.1). 
On the other hand, if $h>1 / \lambda$, then invoke the constraint and set

$$
a=a_{1}=\lambda b(b-c) \sigma^{2} / n+(1-b) \mu+\lambda(1-b)^{2} \tau^{2} ;
$$

that is, set $U G=0$. In this instance,

$$
U G+h B=h B
$$

(6.11) $=h \delta \exp \left\{-\lambda a+\lambda^{2}(b-c)^{2} \sigma^{2} /(2 n)+\lambda(1-b) \mu+\lambda^{2}(1-b)^{2} \tau^{2} / 2\right\}$.

The value $b_{1}$ of $b$ that maximizes $h B$ also maximizes the exponent in the braces in equation (6.11). The maximum of the exponent occurs at

$$
b_{1}=n \tau^{2} /\left(n \tau^{2}+\sigma^{2}\right)=Z .
$$

In this case, $a_{1}=(1-Z) \mu+\lambda \tau^{2}(1-Z)(1-c)$, and we are done. Note that the formula in equation (6.7) is the one we obtain when we maximize $B$ subject to $U G \geq 0$.

We offer the following proposition without proof, thereby sparing the reader of the messy details that are similar to the ones in the proof of Proposition 6.2.

Proposition 6.3: Given the assumptions in Proposition 6.1 and the restrictions $B \geq 1$ and $d(x)=a+b \bar{x}$, we maximize $U G+h B$ when $d=d^{*}$, with $d^{*}$ given by

$$
d^{*}(\boldsymbol{x})=1 / \lambda-h+\{(1-Z) \mu+Z \bar{x}\}+\lambda \tau^{2}(1-Z)(1-c),
$$

for $h \geq 1 / \lambda-(\ln \delta) / \lambda+\lambda \tau^{2}(1-Z) / 2-\lambda c^{2} \sigma^{2} /(2 n)$, and

$$
d^{*}(\boldsymbol{x})=\{(1-Z) \mu+Z \bar{x}\}+(\ln \delta) / \lambda+\lambda \tau^{2}(1-Z)(1-2 c) / 2+\lambda c^{2} \sigma^{2} /(2 n),
$$

for $h$ otherwise.

To illustrate how one might choose the parameter $h$, we offer the following example :

Example: In the normal-normal case, let $\lambda=0.01, \sigma^{2}=250, \mu=1000, \tau^{2}=250$, $\delta=1.5$, and $n=1$. Therefore, $Z=0.50$, so let $c=0.75$. As $h$ increases from 0 to $1 / \lambda=100, U G$ decreases from about 55 to 0 . As $h$ increases beyond 59.375, as determined in Proposition 6.3, $B$ increases from 1 upward.

Suppose we target an underwriting gain of at least 25. The largest value of $h$ is, therefore, 79.571. If we use this value of $h$, then we maximize the relative amount of business $B$, subject to the constraint that $U G \geq 25$. Also, suppose we target a relative amount of business of at least 1.1. The smallest value of $h$ is, therefore, 68.939. If we use this value of $h$, then we maximize the underwriting gain $U G$, subject to the constraint that $B \geq 1.1$. To achieve both $U G \geq 25$ and $B \geq 1.1$, use any value of $h$ between 68.939 and 79.571 . 


\subsection{Optimize Properties of the Book of Business}

An insurance company may set goals concerning the structure of its book of business. We obtain the following lemma that we use to minimize the future grand mean and the future total variance.

Lemma 6.4: Assume the conditions in Theorem 6.1. If the structure of the company's business is given by $\pi(\theta)$ during year 1 , and the credibility premium for year 2 is $a+b x_{1}$, then the expected structure of the business in year 2 is distributed normally with mean $\mu+\lambda \tau^{2}(1-b)$ and variance $\tau^{2}$.

Proof :

$$
\begin{aligned}
\pi_{2}(\theta) & \propto \int p\left(\theta, x_{1}, a+b x_{1}\right) f\left(x_{1} \mid \theta\right) \pi(\theta) d x_{1} \\
& \propto \exp \left\{-\left[\theta^{2}-2 \theta\left(\mu+\lambda \tau^{2}(1-b)\right)\right] /\left(2 \tau^{2}\right)\right\} .
\end{aligned}
$$

It follows that the structure parameter $\Theta$ is expected to be distributed normally with mean $\mu+\lambda \tau^{2}(1-b)$ and variance $\tau^{2}$.

The density $\pi_{2}$ will be independent of the rating parameter $a$ in every case because the term $e^{-\lambda a}$ factors. In the normal-normal case, the distribution is also independent of $c$.

Proposition 6.5: Given the conditions in Lemma 6.4, the grand mean in year 2, expected at time 0 , is minimized when the insurer gives full weight to the policyholder's experience.

Proof: The future grand mean is $\mu_{02}=E_{\theta} E\left[X_{2} \mid \theta\right]=E[\theta]=\mu+\lambda \tau^{2}(1-b)$, in which $0 \leq b \leq 1$. In order to minimize this mean, set $b$ equal to 1 . In other words, give full weight to the policyholder's experience in calculating its second-year premium.

Proposition 6.6: Given the conditions in Lemma 6.4, the variance in year 2, expected at time 0 , is constant, independent of the premium parameters $a$ and $b$.

Proof: The future total variance is

$$
E\left[\left(X_{2}-\mu_{02}\right)^{2}\right]=E_{\theta}\left[\operatorname{Var}\left[X_{2} \mid \theta\right]\right]+\operatorname{Var}_{\theta}\left[E\left[X_{2} \mid \theta\right]\right]=E\left[\sigma^{2}\right]+\operatorname{Var}[\theta]=\sigma^{2}+\tau^{2},
$$

a constant.

\section{POISSON-GAMMA}

To maximize $U G+h B$ in the Poission-gamma case, we apply Theorem 5.1 to obtain the following proposition.

Proposition 7.1: Let $X_{i} \mid \theta \sim P(\theta), i=1, \ldots, n$, be independent and identically distributed Poisson random variables, with unknown mean $\theta$. Let $\Theta \sim G(\alpha, \beta)$ be gamma distributed, with known mean $\alpha / \beta$ and variance $\alpha / \beta^{2}$. Then $U G+h B$ is maximized when 


$$
d^{*}(\boldsymbol{x})=1 / \lambda-h+(\alpha+n \bar{x}) /(\beta+n-\lambda(1-c))
$$

for $\beta+n>\lambda(1-c)$.

Proof: The posterior distribution of $\Theta \mid x$ is gamma, $G(\alpha+n \bar{x}, \beta+n)$. Because $E\left[X_{2} \mid \theta\right]=\theta$, substitute the moment generating function of $\Theta \mid \boldsymbol{x}$ into equation (5.1). The moment generating function of a gamma random variable $\Theta \sim G(\alpha, \beta)$ is

$$
M_{\theta}(t)=E_{\theta}[\exp \{\theta t\}]=\beta^{\alpha} /(\beta-t)^{\alpha},
$$

for $\beta>t$. The derivative, with respect to $t$, of the natural logarithm of this moment generating function is

$$
\alpha /(\beta-t) .
$$

To calculate the credibility formula in equation (5.1), substitute $\alpha+n \bar{x}$ for $\alpha, \beta+n$ for $\beta$, and $\lambda(1-c)$ for $t$ in equation (7.2). We obtain

$$
d^{*}(x)=1 / \lambda-h+(\alpha+n \bar{x}) /(\beta+n-\lambda(1-c)) .
$$

Note that $d^{*}(x)$ is a linear function of the average claim experience $\bar{x}$; therefore, we have a type of exact credibility in this case, as in the normal-normal case. The third term in equation (7.1) is similar to the Bühlmann credibility formula, $(\alpha+n \bar{x}) /(\beta+n)$, except for $-\lambda(1-c)$.

To see how the optimal premium $d^{*}(x)$ changes when the parameter $\lambda$ changes, examine

$$
D \lambda d^{*}(x)=-1 / \lambda^{2}+(1-c)(\alpha+n \bar{x}) /(\beta+n-\lambda(1-c))^{2} .
$$

As in the normal-normal case, observe that if $c$ is sufficiently close to 1 or if $n$ is sufficiently large, then $d^{*}(\boldsymbol{x})$ decreases as $\lambda$ increases; therefore, as the policyholders become more sensitive to the arithmetic difference $\Delta$, the lower the optimal premium will be.

\subsection{Constrained Maximization of $U G+h B$}

As in Section 6.2, we examine two sets of constraints and obtain the following propositions.

Proposition 7.2: Given the assumptions in Proposition 7.1 and the restrictions $U G \geq 0$ and $d(x)=a+b \bar{x}$, we maximize $U G+h B$ when $d=d^{*}$, with $d^{*}$ given by

$$
d^{*}(\boldsymbol{x})=1 / \lambda-h+(\alpha+n \bar{x}) /(\beta+n-\lambda(1-c)),
$$

for $h \leq 1 / \lambda$, and

$$
d^{*}(x)=(\alpha+n \bar{x}) /(\beta+n-\lambda(1-c))
$$

for $h>1 / \lambda$. 
Proof: The moment generating function of a Poisson random variable $P(\theta)$ is

$$
M_{X \mid \theta}(t)=E_{X \mid \theta}[\exp \{x t\}]=\exp \{\theta(\exp \{t\}-1)\},
$$

and similarly

$$
E_{X \mid \theta}[x \exp \{x t\}]=\exp \{\theta(\exp \{t\}-1)\}[\theta \exp \{t\}] .
$$

Use $M_{X \mid \theta}(t)$ and $E_{X \mid \theta}[x \exp \{x t\}]$ to calculate some of the expected values in $U G+h B$. More specifically,

$$
\begin{aligned}
E_{X \mid \theta}[\exp \{\bar{x} t\}] & \left.=M_{X \mid \theta}(t / n)\right\}^{n} \\
& =\exp \{n \theta(\exp \{t / n\}-1)\},
\end{aligned}
$$

and

$$
\begin{aligned}
E_{X \mid \theta}[\bar{x} \exp \{\bar{x} t\}] & \left.=M_{X \mid \theta}(t / n)\right\}^{n-1} E_{X \mid \theta}[x \exp \{x t / n\}] \\
& =\exp \{n \theta(\exp \{t / n\}-1)\}[\theta \exp \{t / n\}] .
\end{aligned}
$$

Also we have for a gamma random variable, $\Theta \sim G(\alpha, \beta)$,

$$
E[\theta \exp \{\theta t\}]=\alpha \beta^{\alpha} /(\beta-t)^{\alpha+1} .
$$

Assume that $\beta>\lambda(1-c)+n(\exp \{-\lambda(b-c) / n\}-1)$, for all $b \in[0,1]$.

Substitute these expressions into $U G+h B$, equation (6.8), to obtain

$$
\begin{aligned}
& U G+h B= \\
& =\delta \exp \{-\lambda a\}(a+h) E[\exp \{\theta(\lambda(1-c)+n(\exp \{-\lambda(b-c) / n\}-1))\}]+ \\
& +\delta \exp \{-\lambda a\} b E[\exp \{\theta(\lambda(1-c)+n(\exp \{-\lambda(b-c) / n\}-1))\} \times \\
& \times[\theta \exp \{-\lambda(b-c / n\}]]-\delta \exp \{-\lambda a\} \times \\
& \times E[\theta \exp \{\theta(\lambda(1-c)+n(\exp \{-\lambda(b-c) / n\}-1))\}] \\
& =\delta \exp \{-\lambda a\}\{(a+h) E[\exp \{\theta(\lambda(1-c)+n(\exp \{-\lambda(b-c) / n\}-1))\}]+ \\
& +(b \exp \{-\lambda(b-c) / n\}-1) E[\theta \exp \{\theta(\lambda(1-c)+ \\
& +n(\exp \{-\lambda(b-c) / n\}-1))\}]\} \\
& =\delta \exp \{-\lambda a\} \beta^{\alpha} /(\beta+n-\lambda(1-c)-n \exp \{-\lambda(b-c) / n\})^{\alpha+1} \\
& \times\{(a+h)(\beta+n-\lambda(1-c)-n \exp \{-\lambda(b-c) / n\}) \\
& +\alpha(b \exp \{-\lambda(b-c) / n)-1\} \text {. }
\end{aligned}
$$

By setting $h=0$ in equation (7.5), we see that

$$
a \geq \alpha(1-b \exp \{-\lambda(b-c) / n\}) /(\beta+n-\lambda(1-c)-n \exp \{-\lambda(b-c) / n\}) .
$$

The values of the parameters $a$ and $b$ that appear in $d^{*}(\boldsymbol{x})$ in equation (7.1) satisfy the inequality (7.6) if and only if $h \leq 1 / \lambda$. Therefore, if $h \leq 1 / \lambda$, then the insurance company does not expect to lose money by using the credibility formula $d^{*}(x)$ in equation (7.1). 
On the other hand, if $h>1 / \lambda$, then invoke the constraint and set $a=a_{1}=\alpha(1-b \exp \{-\lambda(b-c) / n\}) /(\beta+n-\lambda(1-c)-n \exp \{-\lambda(b-c) / n\}) ;$ that is, set $U G=0$. In this instance,

$$
\begin{aligned}
U G+h B & =h B \\
& =h \delta \exp \{-\lambda a\} \beta^{\alpha} /(\beta+n-\lambda(1-c)-n \exp \{-\lambda(b-c) / n\})^{\alpha} .
\end{aligned}
$$

The function $h B$ attains its maximum at

$$
b_{1}=n /(\beta+n-\lambda(1-c)) \text {. }
$$

In this case, $a_{1}=\alpha /(\beta+n-\lambda(1-c))$, and we are done.

We offer the following proposition without providing a proof.

Proposition 7.3: Given the assumptions in Proposition 7.1 and the restrictions $B \geq 0$ and $d(x)=a+b \bar{x}$, we maximize $U G+h B$ when $d=d^{*}$, with $d^{*}$ given by

$$
d^{*}(x)=1 / \lambda-h+(\alpha+n \bar{x}) /(\beta+n-\lambda(1-c)),
$$

for $h \geq 1 / \lambda-(\ln \delta) / \lambda+\alpha /(\beta+n-\lambda(1-c))-$

$-(\alpha / \lambda) \ln \left(\beta /\left(\beta+n-\lambda(1-c)-n \exp \left\{-\lambda\left(b_{1}-c\right) / n\right\}\right)\right)$,

in which $b_{1}=n /(\beta+n-\lambda(1-c))$, and

$$
\begin{aligned}
d^{*}(\boldsymbol{x})= & (\ln \delta) / \lambda+(\alpha / \lambda) \ln \left(\beta /\left(\beta+n-\lambda(1-c)-n \exp \left\{-\lambda\left(b_{1}-c\right) / n\right\}\right)\right) \\
& +n \bar{x} /(\beta+n-\lambda(1-c)) .
\end{aligned}
$$

for $h$ otherwise.

\subsection{Optimize Properties of the Book of Business}

An insurance company may set goals concerning the structure of its book of business. We obtain the following lemma that we use to minimize the future grand mean and the future total variance.

Lemma 7.4: Assume the conditions in Theorem 7.1. If the structure of the company's business is given by $\pi(\theta)$ during year 1 , and the credibility premium for year 2 is $a+b x_{1}$, then the expected structure of the business in year 2 is gamma distributed $G(\alpha, \beta+1-\lambda(1-c)-\exp \{-\lambda(b-c)\})$.

Proof :

$$
\begin{aligned}
\pi_{2}(\theta) & \propto \int p\left(\theta, x_{1}, a+b x_{1}\right) f\left(x_{1} \mid \theta\right) \pi(\theta) d x_{1} \\
& \propto \theta^{\alpha-1} \exp \{-\theta[\beta+1-\lambda(1-c)-\exp \{-\lambda(b-c)\}]\} .
\end{aligned}
$$

It follows that the structure parameter $\Theta$ is expected to be gamma distributed $G(\alpha, \beta+1-\lambda(1-c)-\exp \{-\lambda(b-c)\})$.

Proposition 7.5: Given the conditions in Lemma 7.4, the grand mean in year 2, expected at time 0 , is minimized when the insurer gives full weight to the policyholder's experience. 
Proof: The future grand mean is $\mu_{02}=E_{\theta} E\left[X_{2} \mid \theta\right]=E[\theta]=\alpha /(\beta+1$ $-\lambda(1-c)-\exp \{-\lambda(b-c)\})$, in which $0 \leq b \leq 1$. In order to minimize this mean, set $b$ equal to 1 ; that is, give full weight to the policyholder's experience.

Proposition 7.6: Given the conditions in Lemma 7.4, the variance in year 2, expected at time 0 , is minimized when the insurer gives full weight to the policyholder's experience.

Proof: The future total variance is

$$
\begin{aligned}
E\left[\left(X_{2}-\mu_{02}\right)^{2}\right]= & E_{\theta}\left[\operatorname{Var}\left[X_{2} \mid \theta\right]\right]+\operatorname{Var}_{\theta}\left[E\left[X_{2} \mid \theta\right]\right] \\
= & E[\theta]+\operatorname{Var}[\theta]=\alpha /(\beta+1-\lambda(1-c)-\exp \{-\lambda(b-c)\}) \\
& +\alpha /(\beta+1-\lambda(1-c)-\exp \{-\lambda(b-c)\})^{2} .
\end{aligned}
$$

This variance is minimized when $b$ equals 1 .

\section{FUTURE RESEARCH}

Credibility theory continues to be an important and dynamic area of research in actuarial science as witnessed by the recent work of NORBERG (1992) and PANJER and LI (1994). An aspect of credibility theory that has not been considered very extensively is persistency, which we have addressed here. This work only begins to deal with the problem of credibility and persistency, and we intend to explore this issue further. Some of the outstanding problems are:

\section{Long-term effects}

We have optimized gain functions that span only one year or policy period. Because actuaries consider longer lengths of time, it may be more appropriate to consider the following objectives:

- Maximize the present value of underwriting gain.

- Maximize the stability of the number of insureds; for example, one could minimize the change in the expected number of insureds from year to year.

- Optimize properties of the long-term structure of the book of business.

Another time effect to investigate is the change, or trend, in the number or amount of claims from year to year. Such work could follow the models given by KREMER (1982) or Ledolter, KLUGMAN and LeE (1991).

\section{Different risk sizes and empirical study}

We have not considered different risk sizes. This factor is an important one to include in future models because, in reality, policyholders are not the same size. In future work, we will consider the Bühlmann-Straub model (BÜHLMANN and STRAUB, 1970) and other models that allow for varying risk size (VENTER, 1990), (GoOvaerTs and HoOgSTAD, 1987). 
Such models could be used to fit empirical data in practical research, as in KLUGMAN (1992). An empirical study may also test the validity of the model proposed in this paper with the one mentioned at the end of Section 4.

\section{ACKNOWLEDGEMENTS}

I thank my colleagues and friends for graciously giving their time to help me organize my thoughts and this paper more clearly: Jed Frees, Jim Hickman, and David Li. I also thank two anonymous referees for suggesting valuable changes.

\section{REFERENCES}

BÜhlmann, H. (1967) Experience rating and credibility. ASTIN Bulletin 4, 199-207.

Bühlmann, H. (1970) Mathematical Models in Risk Theory. Springer-Verlag, New York.

Bühlmann, H. and Straub, E. (1970) Glaubwürdigkeit für Schadensätze. Mitteilungen der Vereinigung Schweizerischer Versicherungs-Mathematiker 70, 111-133.

Gerber, H.U. (1980), Credibility for Esscher premiums. Mitteilungen der Vereinigung Schweizerischer Versicherungs-Mathematiker 80, 307-312.

Goovaerts, M.J. and Hoogstad, W. J. (1987) Credibility Theory. Surveys of Actuarial Studies, No. 4, Nationale-Nederlanden, Rotterdam, The Netherlands.

JEWELl, W.S. (1974a) Credibility is exact Bayesian for exponential families. ASTIN Bulletin 8, 77-90.

JEWELL, W. S. (1974b) Regularity conditions for exact credibility. ASTIN Bulletin 8, 336-341.

Klugman, S. A. (1992) Bayesian Statistics in Actuarial Science with Emphasis on Credibility. Kluwer Academic Publishers, Boston.

Kremer, E. (1982) Credibility theory for some evolutionary models. Scandinavian Actuarial Journal, $129-142$.

Ledolter; J., Klugman, S. and LeE, C.S. (1991), Credibility models with time-varying trend components, ASTIN Bulletin, $21: 73-86$.

MorewoOd. F. G. (1992) Planning and control issues. In Group Insurance (ed. W. F. Bluhm), chapter 34, ACTEX Publications, Winsted, Connecticut.

NORBERG, R. (1992) Linear estimation and credibility in continuous time. ASTIN Bulletin 22, 149-165.

PANJER, H.H. and LI, D.X. (1994) Credibility models: An estimating function approach. Working paper.

SunDT, B. (1983) Credibility models allowing durational effects. Mitteilungen der Vereinigung Schweizerischer Versicherungs-Mathematiker 83, 63-87.

TAYLOR, G.C. (1975) Credibility under conditions of imperfect persistency. In Credibility: Theory and Applications (ed. P. M. Kahn), pp. 391-400, Academic Press, New York.

Venter, G. (1990) Credibility. In Foundations of Casualty Actuarial Science, Casualty Actuarial Society, New York.

Willmot, G.E. (1994) Introductory Credibility Theory. Institute of Insurance and Pension Research, University of Waterloo, Waterloo, Ontario.

VIRGINIA R. YOUNG

School of Business, Grainger Hall

University of Wisconsin-Madison

Madison, WI, USA 53706 